\title{
Inclusión educativa en tiempos de COVID-19: Uso de redes sociales en personas con discapacidad intelectual
}

\section{(Educational Inclusion in Times of COVID-19: Use of Social Media for People with Intellectual Disabilities)}

\author{
Mónica Bonilla-del-Río \\ Universidad de Huelva (España) \\ María Luisa Sánchez Calero \\ Universidad Complutense de Madrid (España)
}

DOI: https://doi.org/10.5944/ried.25.1.30875

\section{Cómo referenciar este artículo:}

Bonilla-del-Río, M., y Sánchez Calero, M. L. (2022). Inclusión educativa en tiempos de COVID-19: Uso de redes sociales en personas con discapacidad intelectual. RIED. Revista Iberoamericana de Educación a Distancia, 25(1), pp. 141-161. https://doi.org/10.5944/ried.25.1.30875

\section{Resumen}

Los procesos de enseñanza-aprendizaje se han transformado de manera repentina e irremediable debido a las consecuencias provocadas por la COVID-19. Tanto los educadores como el alumnado se han visto forzados a adaptarse a las imprevistas necesidades contextuales y pedagógicas, recurriendo a los dispositivos y entornos virtuales para desarrollar sus prácticas didácticas. Estos retos en ocasiones han provocado ciertas dificultades, pero también nuevas oportunidades de comunicación y de acceso a la información y el conocimiento. En la presente investigación se analizan los usos de las plataformas digitales por parte tanto de los jóvenes con discapacidad como de los especialistas y familias de un centro de la Comunidad de Madrid para hacer frente a esta nueva realidad. Para ello, se ha aplicado un cuestionario y se han realizado entrevistas semiestructuradas a los diferentes agentes educativos. Los resultados inciden en los beneficios y barreras que se han encontrado durante el proceso de adaptación a la modalidad virtual impuesta de forma abrupta por la pandemia, así como en los cambios que el confinamiento ha supuesto en el uso que hacen de los dispositivos y plataformas digitales. Las conclusiones apuntan a que hoy más que nunca la inclusión digital implica la inclusión social y educativa. Sin embargo, si no se apuesta por ella estaríamos ante nuevas formas de exclusión que se pronuncian en este contexto de emergencia sanitaria y distanciamiento social. Por tanto, la educación mediática se establece como un factor clave para evitar que se incremente la brecha digital en este colectivo. 
Palabras clave: educación inclusiva; redes sociales; diversidad; inclusión; educación especial; competencia mediática.

\begin{abstract}
Teaching-learning processes have been suddenly and irremediably transformed by the consequences of COVID-19. Both educators and learners have been forced to adapt to unforeseen contextual and pedagogical needs, using virtual devices and environments to develop their didactic practices. These challenges have sometimes led to certain difficulties, but also to new opportunities for communication and access to information and knowledge. This research analyses the uses of digital platforms by both young people with disabilities as well as the specialists and families of a centre in the Region of Madrid to deal with this new reality. To this end, a questionnaire was applied and semi-structured interviews were conducted with different educational agents. The results highlight the benefits and barriers encountered during the process of adapting to the virtual modality abruptly imposed by the pandemic, as well as the changes that confinement has brought about in the use of digital devices and platforms. The conclusions point to the fact that today more than ever, digital inclusion implies social and educational inclusion. However, if we do not support it, we would be facing new forms of exclusion that are pronounced in this context of health emergency and social distancing. Therefore, media education is established as a key factor to avoid an increase in the digital gap among this group.
\end{abstract}

Keywords: inclusive education; social media; diversity; inclusion; special education; media competence.

Las dificultades generadas por la COVID-19 en todos los niveles y a escala mundial, así como el confinamiento derivado por la emergencia sanitaria, provocaron que las instituciones educativas tuvieran que reaccionar y adaptarse de manera vertiginosa a las nuevas demandas de la sociedad. La modalidad virtual se instauró como una necesidad imperiosa en los procesos de enseñanza-aprendizaje (Martínez-Garcés y Garcés-Fuenmayor, 2020), impuesta por el cierre de los centros escolares a raíz de la declaración del estado de alarma. Esta crisis sanitaria ha provocado la mayor disrupción histórica en los sistemas educativos, provocando que casi 1.600 millones de estudiantes de todo el mundo se viesen afectados por esta situación (UNESCO, 2020). Estas estadísticas suponen que el $94 \%$ de la población estudiantil, de más de 190 países y de todos los continentes, sufriese los estragos del cierre de las escuelas y otros espacios de aprendizaje. La relevancia adquirida por la educación online durante este período fue un hecho sin precedentes, que supondrá un antes y un después en las prácticas e instituciones educativas (Expósito y Marsollier, 2020). Ante este contexto, los soportes digitales se establecieron como un factor fundamental, lo que provocó que la brecha de acceso se incrementase por la pandemia, ya que disminuyeron las posibilidades de accesibilidad a los recursos digitales y, por tanto, al aprendizaje por parte de las poblaciones vulnerables (García-Aretio, 2021). 
Es innegable que la falta de recursos, de acceso a internet o el desconocimiento hacia el uso de las plataformas digitales puede generar desigualdades para una parte del alumnado y sus familias. Estas dificultades pueden estar causadas por diversos motivos como pueden ser los factores económicos, sociales o geográficos (CorralOllero y de-Juan-Fernández, 2021). Esta situación supuso, y sigue suponiendo, un reto para todos los agentes educativos.

En esta línea, hoy más que nunca, Internet se ha convertido en una herramienta sustancial en todos los ámbitos de la vida, entre ellos el educativo. Pero a pesar de ser una parte integral de la cotidianidad de las personas, especialmente de los jóvenes, existe cierta falta de conocimiento sobre el uso que hacen de Internet las personas con discapacidad (Alfredsson-Ågren et al., 2020). Es cierto que a nivel legislativo se están logrando avances en la promoción e implantación de normas para mejorar la accesibilidad de las personas con discapacidad, entendiendo este factor como un medio fundamental para alcanzar la inclusión y participación plena de este colectivo en la sociedad y garantizar sus derechos. Podría destacarse, en este caso, la adopción de medidas para promover el acceso de las personas con discapacidad a las tecnologías, Internet y los sistemas de información y comunicación (Tejada-Ríos, 2021).

De este modo, la formación virtual impartida en el ámbito de la educación especial ha de adaptarse no solo al nuevo contexto, sino también a las características, necesidades e intereses de las personas con discapacidad. La educación a distancia puede suponer una oportunidad para apoyar el aprendizaje de las personas con necesidades educativas especiales, siempre y cuando se proporcione tecnología accesible, información necesaria y apoyos (Karasel et al., 2020). Sin embargo, si no se dan estas circunstancias, tal y como expone Martín-Blanco (2021), puede provocar situaciones de exclusión, que se ven agravadas de manera exponencial por las consecuencias de la COVID-19 y el estado de excepcionalidad que limita o reduce las sesiones presenciales.

En este sentido, tal y como ponen de manifiesto Moreno-Rodríguez et al. (2020), en el caso del alumnado con discapacidad la situación se agravó aún más, debido a que a las dificultades de aprendizaje previas a la teleeducación forzada, se sumaron las causadas por la no presencialidad y la utilización de las TIC como recurso único para el desarrollo de los procesos de enseñanza-aprendizaje, lo que ha podido provocar situaciones de exclusión por la falta de acceso a una educación inclusiva y de calidad, la falta de recursos y de apoyos suficientes.

En este escenario, los docentes han de enfrentarse a nuevos desafíos, potenciando al máximo sus habilidades sociales y digitales y adecuando sus prácticas pedagógicas al entorno virtual a través de herramientas como plataformas de videoconferencia (Cáceres-Piñaloza, 2020) y las redes sociales para mantener el contacto con el alumnado o sus familias. En este sentido, el profesorado ha tenido que emplear recursos que facilitasen la comunicación y permitiesen la propuesta y desarrollo de actividades a distancia a través de diferentes vías como, por ejemplo: 
Realización de sesiones virtuales mediante plataformas como Zoom, Google Meet o Microsoft Teams; búsqueda de canales alternativos de comunicación como grupos de WhatsApp o redes sociales; uso de recursos colaborativos como Google Drive; y el aprovechamiento de repositorios, plataformas o vídeos educativos (Arce-Peralta, 2020). Algunos de los inconvenientes más pronunciados a los que se han visto sometidos los especialistas en educación especial son la dificultad de ofrecer una atención de calidad y personalizada a través de plataformas virtuales y la suspensión del apoyo profesional especializado que ofrecían (Murillo y Duk, 2020), que en numerosas ocasiones no es posible realizar a distancia o de manera telemática. Otra de las dificultades se basa en el uso limitado que hacía el profesorado de los recursos digitales y mediáticos, que se acentúa en ambientes de formación virtual y que habitualmente está vinculado a la motivación y a su desconocimiento para emplearlos de manera provechosa y exitosa en las prácticas educativas (Martínez-Garcés y Garcés-Fuenmayor, 2020). Tal y como ponen de manifiesto diversas investigaciones en este ámbito, el profesorado podría advertir limitaciones respecto a su nivel de competencia mediática al haber hecho un uso más esporádico e instrumental de las tecnologías (Aguaded et al., 2021).

Asimismo, en el caso de las personas con discapacidad el rol de la mediación parental juega un papel muy importante. De acuerdo con Wright et al. (2021), el apoyo familiar hace referencia a la percepción de las personas al sentir cuidado, respeto y valoración por parte de los miembros de su familia, destacando este apoyo como un factor fundamental en la adolescencia, debido a los grandes cambios y riesgos a los que se enfrentan los jóvenes en el ámbito social y emocional durante esta etapa, así como en su desarrollo en general. La importancia del rol de las familias se ha acentuado, aún más si cabe, durante el período pandémico. De acuerdo con Karasel et al. (2020), el apoyo familiar ha sido clave para que las prácticas educativas pudieran desarrollarse en modalidad virtual, siendo este agente educativo un apoyo necesario para los docentes a la hora de facilitar los procesos de enseñanza-aprendizaje en el ámbito de la educación especial. Sin embargo, según Albalá y Guido (2020), el funcionamiento telemático también permitió comprobar las carencias a nivel tecnológico y socioeducativo para algunas familias de España, poniendo en evidencia la desigualdad de oportunidades y la brecha social, digital y educativa que se incrementa en los hogares más desfavorecidos, debido a la falta de recursos materiales, sociales y culturales, que contribuyen al acceso desigual a las plataformas digitales de aprendizaje y perjudican la continuidad en la formación de los jóvenes. La alfabetización mediática, por consiguiente, se instaura como un derecho básico de la ciudadanía y como una necesidad de los sistemas educativos del siglo XXI, que contribuiría a la reducción o eliminación de las barreras que dificultan la usabilidad y accesibilidad de las personas con discapacidad a las tecnologías (Bonilla-del-Río et al., 2018).

Considerando lo expuesto anteriormente, el presente estudio tiene como objetivo general analizar los beneficios y barreras que encuentran las personas con 
discapacidad intelectual en el uso de las plataformas digitales y comprobar cómo ha afectado la pandemia de la COVID-19 en este ámbito a los diferentes agentes educativos de un centro de educación especial. Como objetivos específicos se plantea analizar: 1) El nivel de utilización de las redes sociales por parte de las personas con discapacidad; 2) Los aspectos que condicionan el uso efectivo de las redes sociales por parte del colectivo; 3) La frecuencia de uso y cuáles son las plataformas digitales más empleadas; 4) La percepción de los agentes educativos ante el uso de las redes sociales, así como los beneficios y barreras principales ante el uso de estas plataformas; y 5) En qué aspectos ha afectado la COVID-19 tanto en los procesos de enseñanza-aprendizaje como en la vida personal de este colectivo en relación con el uso de estas plataformas.

\section{METODOLOGÍA}

La investigación se centra en un estudio de caso al focalizarse en el análisis de la realidad de un contexto educativo concreto, mediante la realización de una descripción contextualizada y la revelación de las relaciones que se producen en esta institución educativa y la situación particular derivada por la COVID-19 (ÁlvarezÁlvarez y San-Fabián-Maroto, 2012).

\section{Instrumentos}

Los instrumentos de investigación consisten en un cuestionario sobre el uso inclusivo de las redes sociales, cuya aplicación se realizó a través de Google Forms, y en entrevistas semiestructuradas.

El cuestionario se aplicó durante los meses de enero y abril, informando a los participantes sobre el estudio y solicitando su aceptación y consentimiento para la participación en la investigación. Se divide en tres grandes secciones, que incluyen:

- Datos sociodemográficos: En primer lugar, se les solicito información sobre su rol dentro de la institución educativa, su edad, el género con el que se identifican y su nivel educativo.

- Nivel de utilización de las redes sociales: Se les preguntó sobre su percepción acerca de los factores que, bajo su criterio, condicionan el uso de las redes sociales por parte de las personas con discapacidad. Se incluyeron cuatro tipos de factores que podrían influir en el uso de las redes sociales por parte de este colectivo: autonomía personal y conocimiento de las redes sociales, apoyo, accesibilidad y barreras. El primero midió hasta qué punto los agentes educativos consideran que el grado de independencia de las personas con discapacidad, así como sus aptitudes físicas o psicosociales y su conocimiento sobre estas plataformas digitales influyen en el nivel de uso que hacen de las mismas. El apoyo valoró 
cuestiones como la dependencia de la aprobación familiar o del profesorado, la sensibilidad de las personas de su entorno familiar, educativo o de amistades en relación con la promoción del uso de las redes sociales (interés, prejuicios, apoyo...), así como el apoyo y motivación para el uso de las redes sociales desde el ámbito familiar. La accesibilidad hace hincapié en la posesión de terminal de acceso, la accesibilidad económica o tecnológica en el entorno familiar y el lugar de acceso y utilización de estos recursos, bien sea en el centro educativo o en el familiar. Por último, las barreras incluyeron las dificultades de accesibilidad y falta de ayudas económicas (complejidad para usar la propia plataforma, falta de adaptaciones o de recursos con las prestaciones adecuadas a las necesidades de la persona con discapacidad...) y la falta de ayudas técnicas (obstáculos o falta de apoyo en el uso eficiente o en la resolución de problemas derivados de su uso...). Se les presentó una escala Likert de 10 puntos, en la que 1 se corresponde con "nada condicionado" y 10 es "extremadamente condicionado".

Además, se incluyeron tres preguntas más con opción de respuesta múltiple acerca de su percepción sobre la cantidad de personas con discapacidad del centro que emplean las redes sociales, las plataformas qué más emplean y los motivos que les impulsan a su utilización.

- Percepción sobre el uso de redes sociales: En esta sección se presentaron preguntas con opción de respuesta múltiple, respuestas dicotómicas y dos preguntas abiertas para que los participantes pudieran explicar más en profundidad su opinión respecto al uso de las redes sociales. Los ítems contemplan su postura ante el uso de estas plataformas digitales por parte de las personas con discapacidad, los beneficios que consideran que tienen este tipo de recursos para este colectivo, la frecuencia de uso de las redes sociales por parte de las personas con discapacidad, la importancia que otorgan a la formación de las personas con discapacidad ante el uso de estos recursos y finalmente a su percepción sobre el cambio que ha podido suponer la COVID-19 en el uso de las redes sociales por parte de los jóvenes con discapacidad.

Las entrevistas semiestructuradas se realizaron en el mes de abril y estuvieron compuestas por doce preguntas. Se procedió a su validación a través del juicio de expertos (2), cuya opinión y propuestas de mejora se tuvieron en cuenta para la construcción del instrumento final. A pesar de que en un primer momento el estudio se planteó para analizar el uso específico de las redes sociales, se decidió sustituir este término durante las entrevistas por "plataformas digitales", ya que en el cuestionario los participantes destacaron el uso de recursos de plataformas de videoconferencia como Zoom. En este sentido, este término engloba tanto las redes sociales como otras plataformas digitales que favorecen los procesos comunicativos y de socialización. 


\section{Muestra}

La muestra estuvo conformada por 39 personas, adultas, pertenecientes al centro ocupacional de AMI-3 situado en la localidad madrileña de Tres Cantos. Asociación fundada en 1.992 por un grupo de padres que tenían algún hijo/a con algún tipo de discapacidad y siendo su principal objetivo desde hace veintiocho años proporcionar atención a las familias con personas con discapacidad intelectual. Esta asociación se halla inscrita en el Registro provincial de Asociaciones, siendo reconocida como entidad de Acción Social y servicios sociales.

La selección de los participantes de este estudio se realizó a través del muestreo por conveniencia. Y los individuos participantes en la presente investigación han sido trabajadores del propio centro ocupacional, usuarios y las familias, padres o tutores.

El cuestionario fue respondido por un total de 32 participantes, 16 de ellos familiares y los otros 16 agentes educativos del centro, entre los que destacan roles como educadores, voluntarios, psicólogos y monitores de ocio. El 81,3\% son mujeres y el 18,7\% hombres. La edad de los participantes oscila entre los 21 y los 72 años, siendo el promedio de 47,53. Una muestra de pequeña dimensión justificando y teniendo en cuenta las propias características de AMI-3. Un centro que solo dispone de 38 plazas COFOIL (Centro ocupacional de Formación e Inserción Laboral), con 23 profesionales en plantilla y 100 familias como socios.

En las entrevistas participaron siete personas, con diferentes roles dentro del centro. En este sentido, se entrevistó a un familiar, una monitora de ocio, una psicóloga, una coordinadora del centro y tres jóvenes con discapacidad usuarias en la institución, con edades comprendidas entre los 30 y 40 años.

\section{RESULTADOS}

En primera instancia, se muestran los resultados más significativos obtenidos en el cuestionario cumplimentado por los profesionales del centro y las familias. En relación al nivel de utilización de las redes sociales por parte de las personas con discapacidad, se solicitó a los agentes educativos que valorasen mediante una escala de tipo Likert, donde 1 es "Nada condicionado" y 10 es "Extremadamente condicionado", de qué manera consideraban que influyen los siguientes aspectos en la utilización de estos recursos por parte este colectivo (Figura 1).

Entre los aspectos que los agentes educativos consideran como más influyentes, mediante la selección de la opción de mucho o bastante, cabe destacar las aptitudes físicas o psicosociales (65,7\%), así como la sensibilidad de las personas de su entorno a la hora de promover el uso efectivo de las redes sociales $(56,3 \%)$, lo que pone de manifiesto la importancia que le otorgan, por un lado, a las propias aptitudes de las personas con discapacidad, pero también a los estímulos, motivación y apoyos que reciben en su entorno más cercano para el impulso de la utilización de este tipo de 
recursos por parte del colectivo. En este sentido, la mitad de los encuestados (50,1\%), consideran que la accesibilidad económica o tecnológica en el ámbito familiar influye mucho o bastante a la hora de que las personas con discapacidad hagan un uso efectivo de estas plataformas digitales, situándose como el tercer elemento más influyente para los participantes encuestados.

\section{Figura 1}

Condicionamiento del uso efectivo de las redes sociales por parte de las personas con discapacidad

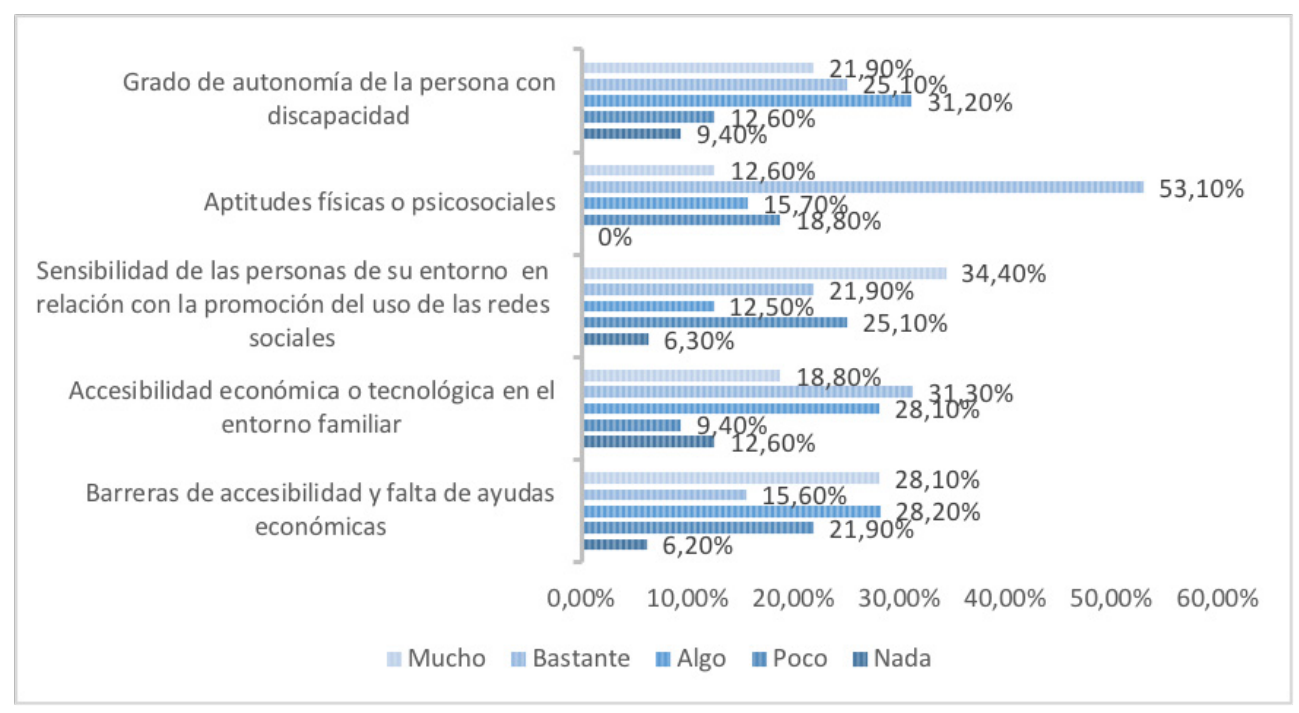

Además de estos parámetros, los datos en relación con el "Lugar de acceso" son significativos, ya que más de la mitad de los participantes consideran que el acceso desde el ámbito familiar está muy condicionado (56,3\%), mientras el porcentaje disminuye con respecto a la importancia que le otorgan al acceso a las redes sociales desde el entorno educativo (37,5\%). En lo referente al "Apoyo y motivación para el uso de las redes sociales", los resultados muestran porcentajes más similares, ya que un $62,7 \%$ de los encuestados consideran que se trata de un factor muy condicionante en el ámbito familiar y un 59,4\% en el ámbito educativo.

En cuanto al nivel de utilización de las redes sociales, no existe consenso entre los agentes educativos acerca del número de jóvenes con discapacidad que acceden a este tipo de recursos. El porcentaje más alto corresponde a la percepción de los profesionales y familiares que manifiestan que "la mayoría de jóvenes con discapacidad del centro acceden a las redes sociales" $(28,1 \%)$, mientras que el más bajo hace alusión a que "ningún o casi ningún joven con discapacidad usa o accede a 
las redes sociales" (3,1\%). Sin embargo, el $25 \%$ destaca que, aunque no representan la mayoría, más de la mitad de los jóvenes del centro acceden a las redes sociales, el $21,9 \%$ considera que solo una parte hace uso de estos recursos y el $21,9 \%$ declara no disponer de esta información.

Sobre la frecuencia de uso, el 50\% exponen que los jóvenes con discapacidad del centro las emplean a diario, el $43,8 \%$ casi todos los días de la semana y tan solo el $6,3 \%$ exponen que de manera puntual algunos días de la semana. Sobre el tiempo de uso, el 59,4\% señalan que entre una y dos horas al día, el 28,1\% más de dos horas al día y el 12,5\% menos de una hora al día. Ningún participante escoge las opciones de "nunca las utilizan" en estos ítems.

Las redes más empleadas, tal y como se puede apreciar en la figura 2, son WhatsApp (90,6\%), seguida de YouTube $(59,4 \%)$ e Instagram $(28,1 \%)$ y Facebook $(15,6 \%)$. También destacan, a pesar de no ser considerada una red social genérica, el uso del servicio de videoconferencia Zoom (12,5\%).

\section{Figura 2}

Redes sociales más empleadas por parte de las personas con discapacidad

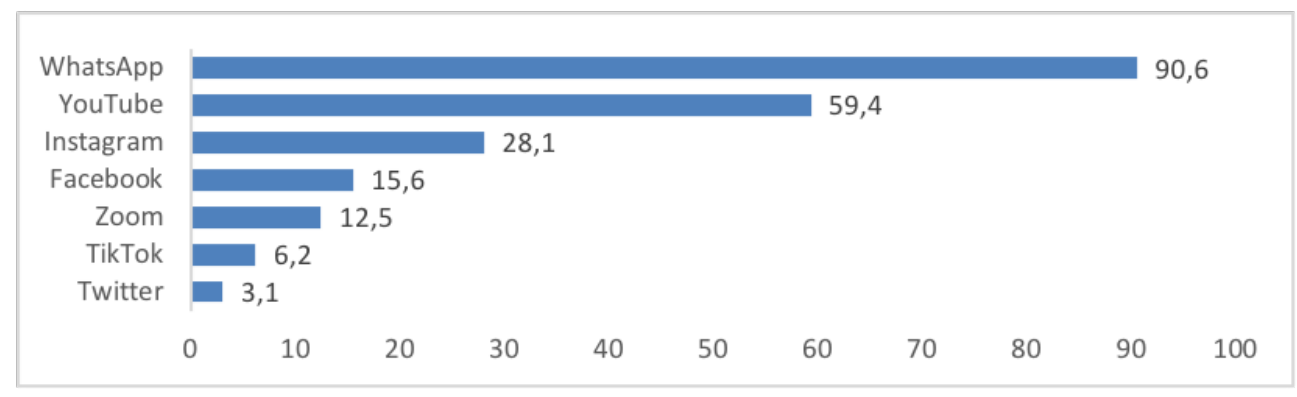

Los motivos que más impulsan el uso de las redes sociales, según los participantes del estudio, son (Figura 3): la necesidad de comunicarse con amigos (93,8\%), la familia $(59,4 \%)$ y los docentes $(43,8 \%)$, así como el entretenimiento $(78,1 \%)$. 


\section{Figura 3}

Principales usos de las redes sociales por parte de las personas con discapacidad

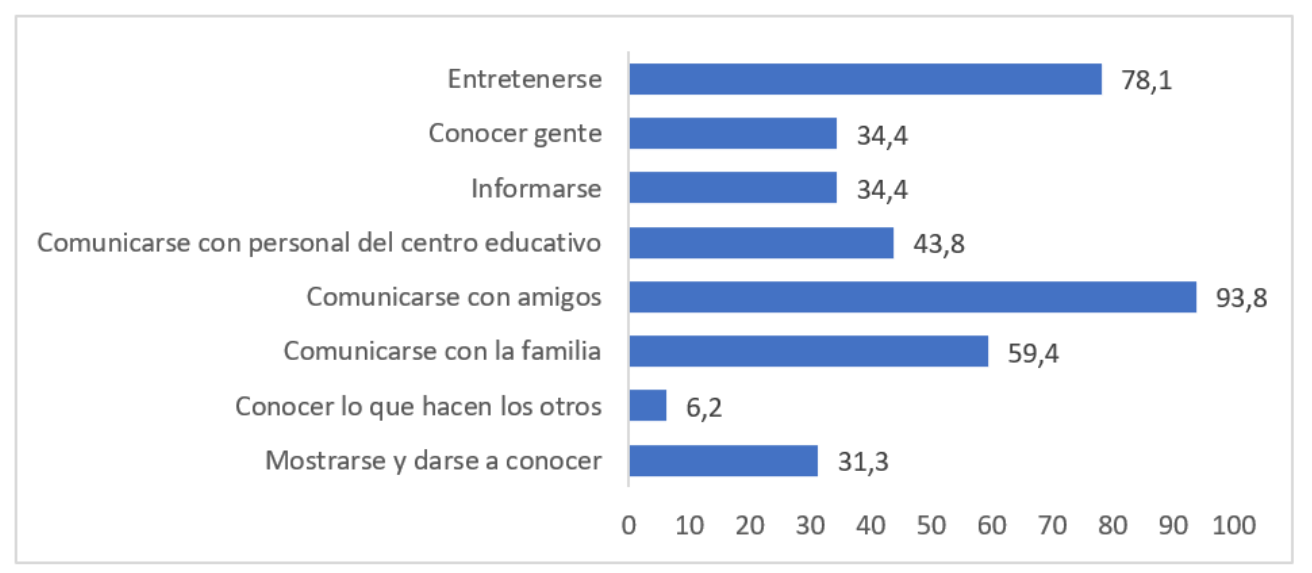

Por otro lado, cabe mencionar que los agentes educativos otorgan gran relevancia al uso de las redes sociales por parte de los jóvenes con discapacidad, mostrando una percepción positiva hacia el uso de estos recursos. En esta línea, el 59,4\% consideran que las redes sociales favorecen la integración de los jóvenes y mejoran las relaciones interpersonales con otros; el 50\% indican que les parece importante apoyar a sus hijos o alumnos a que participen en estos espacios digitales y el 43,8\% declaran hacer efectivo este apoyo. Asimismo, exponen la necesidad que otorgan a la supervisión del uso que hacen de las redes sociales, ya que el 46,9\% manifiesta permitir el uso de las redes sociales siempre y cuando pueda controlar y supervisar su actividad. En cambio, tan solo el 12,5\% prefiere que sus hijos o alumnos no se expongan en redes sociales y únicamente el $6,3 \%$ indican que el uso de las redes no es saludable y les perjudicaría.

Como beneficios más destacados (Figura 4), las familias y docentes coinciden en que las redes sociales mejoran las relaciones de los jóvenes con discapacidad con amigos y compañeros (75\%) y les permiten entretenerse y estar informados $(71,9 \%)$. Mientras que únicamente el 3,1\% considera que estos recursos no ofrecen ningún tipo de ventajas para este colectivo. 


\section{Figura 4}

Beneficios del uso de las redes sociales para las personas con discapacidad

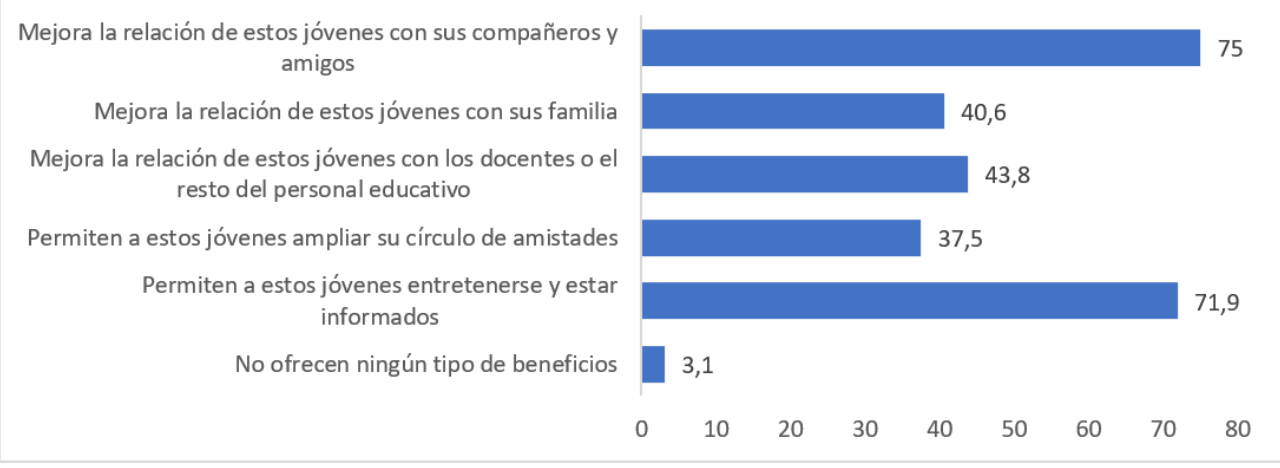

Por su parte, el 90,6\% considera que el uso de las redes sociales es un elemento de motivación de los jóvenes dentro del grupo y todos los participantes consideran útil la capacitación de los jóvenes con discapacidad hacia el uso de las redes con respecto a su desarrollo personal. Entre las razones más destacadas que justifican sus respuestas, los participantes exponen que la formación en este ámbito es necesaria para que los jóvenes con discapacidad puedan hacer un uso efectivo de estas plataformas y puedan evitarse o disminuirse las situaciones de riesgo; para poder sentirse integrados en la sociedad y comunicarse con otras personas; y porque son recursos necesarios en la actualidad que pueden favorecer, además, su autonomía.

Del mismo modo, todos los encuestados consideran que la situación derivada de la COVID-19 y el consecuente confinamiento ha supuesto un impulso en el uso de las redes sociales entre los jóvenes con discapacidad. Entre las razones que han motivado que aumentase la utilización de estos recursos durante la pandemia destacan: la falta de comunicación personal debido a la reducción de relaciones presenciales; el uso de aplicaciones en clases online, que les ha acercado a estas herramientas y les mantiene conectados, promoviendo una disminución del aislamiento social y el miedo a la situación; y la necesidad de buscar alternativas de comunicación y entretenimiento.

A continuación, se exponen los datos de las siete entrevistas realizadas a profesionales, gestores, pedagogos, psicólogos, familias e incluso a tres personas con discapacidad intelectual, cuyos resultados ponen de manifiesto las necesidades y principales dificultades con las que se han encontrado durante la pandemia en el uso de las plataformas digitales, apuntando, desde su experiencia, a las formas más factibles para resolverlas.

Sin duda, en estas plataformas han encontrado un gran aliado para comunicarse, pero también se observan, tal y como afirman pedagogos y formadores, las barreras más frecuentes a las que se enfrentan. Así, mediante la realización de doce preguntas, 
reestructuradas para este análisis en diferentes temas, se han recogido resultados que abordan aspectos como: las plataformas digitales más utilizadas, el uso que les han dado, las dificultades más habituales encontradas en su manejo, y la finalidad en el uso de las mismas que se plantean en los colectivos de personas con discapacidad intelectual.

¿Considera que la pandemia de la COVID-19 ha supuesto un cambio sustancial en el uso que las personas con discapacidad intelectual hacen de las plataformas digitales?

Los entrevistados en este estudio coinciden en la totalidad de las respuestas en que la pandemia ha supuesto un gran cambio en este sentido. Los técnicos, los profesionales y los usuarios con discapacidad entrevistados declaran que les ha afectado de manera muy significativa sobre todo a la hora de comunicarse, pero también manifiestan que ha existido una brecha en cuanto al acceso y uso de Internet. De este modo, según los entrevistados, son las posibilidades de acceso a Internet, la causa que ha dividido a los participantes en dos grupos diferenciados. El primero, constituido por un porcentaje mayor de usuarios, reconoció que sí tenían acceso a la tecnología, así como la competencia digital necesaria para utilizarla correctamente por ellos mismos o con la ayuda facilitada por su familia. Mientras que el segundo grupo sí que, a juicio de los profesionales, mostró necesidades de formación y carencias en el acceso y uso de Internet.

Por tanto, los resultados indican que el cambio sustancial no ha estado tanto en determinar el buen uso o acogida que han tenido estas plataformas por parte de los participantes, sino en las dificultades de utilización que ha tenido un grupo menor.

Asimismo, constatan que se ha producido una brecha a nivel formativo, dado que los profesionales han tenido que apoyar a algunos usuarios y familiares mediante la creación de tutoriales que luego han compartido a través de vídeos de YouTube e incluso señalan que durante y después del confinamiento se han realizado acompañamientos individuales. Las diferencias más notables se encontraron en función de la edad, resultando más fácil y determinante el mayor uso, accesibilidad y manejo de este tipo de plataformas y servicios entre los usuarios más jóvenes del Centro.

Una formación pedagógica que ha resultado constructiva para todos los implicados en el proceso -familias, profesionales, pedagogos, psicólogos, voluntarios y gestores- y que se ha constituido como el elemento clave para adquirir las competencias necesarias en el manejo de las plataformas digitales. Así, mientras los profesionales hablan del empeño del Centro por apoyar formativamente a familias y usuarios para tratar de suplir estas carencias, las familias responden que no siempre ellas mismas tenían la competencia digital necesaria o los equipos apropiados para hacer un buen uso de las plataformas. En este sentido, a pesar de que no han sido muchos los casos, sí que algunas familias han precisado de donaciones de equipos 
informáticos o de formación concreta, contestan desde la gestión del Centro. Según expresa la coordinadora de proyectos del Centro, son pocos los usuarios que no tenían acceso a la tecnología y en estos casos se consiguieron patrocinadores y voluntarios informáticos para instalarlos. Una cuestión que no ha afectado principalmente a los usuarios del Centro Ocupacional de AMI-3, teniendo en cuenta que la renta bruta media en el municipio de Tres Cantos se situó en 2018 en 42.248 euros según datos actualizados en octubre de 2020.

Por su parte, las familias hacen hincapié en la necesidad de promover el "uso correcto" o "uso responsable" de las herramientas o canales de comunicación. Una desconfianza por parte de los familiares que, según los profesionales del Centro, existe y que únicamente se resuelve con formación y vigilancia apropiada, creando talleres formativos que ayudan a solventar estos problemas.

¿Cuáles han sido las plataformas digitales más empleadas, con qué frecuencia las han utilizado y en qué tipo de actividades han resultado más útiles?

En línea con los resultados obtenidos en el cuestionario y expresando los resultados en términos de porcentajes, según los participantes en las entrevistas -gestores, profesionales, educadores, psicólogos, o familias-, puede afirmarse que en un $100 \%$ de sus respuestas reconocen que las plataformas más empleadas por los usuarios del Centro han sido: WhatsApp, Zoom, YouTube, Facebook, TikTok e Instagram. Además, sostienen que se les ha otorgado un uso distinto a cada una de ellas y que también se aprecian diferencias significativas en cuanto al tiempo de uso por parte de los usuarios del Centro, aunque en general señalan que las utilizan diariamente y una media de hasta dos horas al día.

Los usuarios con discapacidad del Centro que participaron en las entrevistas reconocen que las utilizan principalmente con el objetivo de establecer comunicación con sus amigos, familiares o con los formadores del Centro, haciendo una escasa alusión a motivos como la formación o el entretenimiento. Este último aspecto contrasta con el resultado obtenido en los cuestionarios y la percepción que los agentes educativos manifestaban sobre el uso de las redes sociales por parte de las personas con discapacidad con respecto al entretenimiento, ya que lo consideraban como uno de los principales usos de las redes sociales por parte del colectivo con un $78,1 \%$. Sin embargo, uno de los tres entrevistados comentó que lo han utilizado para mostrarse o darse a conocer, coincidiendo estas respuestas con aquellos que han potenciado principalmente el uso de YouTube o Instagram.

Las respuestas de los formadores y de las familias apuntaban a cierto nivel de "desconfianza", exponiendo que son muchos los aspectos que les preocupan sobre el uso de estas plataformas, entre los que destacan "el miedo a la invasión de la intimidad y la generación de conflictos entre ellos”, tal y como manifestaba una de las pedagogas entrevistadas. Una opinión también compartida por el padre entrevistado, que aseguraba: "Mis miedos no se centran tanto en permitirles el uso 
de "chats", aplicaciones de mensajería instantánea o redes sociales, sino en poder controlar y supervisar lo que hacen en estas plataformas digitales y las aplicaciones que instalan".

Una respuesta que corroboran los profesionales del Centro entrevistados que mencionan la existencia de "desconfianza" y "reticencia" que en ocasiones existe por parte de las familias en el uso de las redes sociales, resaltando que resulta más frecuente cuando el grado de discapacidad del usuario es menor y por tanto muestra mayor independencia en el uso de este tipo de aplicaciones.

En este sentido, las declaraciones de los usuarios con discapacidad atendían a que la elección de una u otra plataforma se debe principalmente a las características y funciones que ofrecen, ya que priorizan la facilidad de manejo y que puedan comunicarse entre ellos sin diferencias y según sus habilidades. "Que tenga imagen o que pueda grabar un audio", son los elementos preferidos para elegir una u otra plataforma, según comenta una de las usuarias del Centro entrevistadas, que admite que es la funcionalidad que lo convierte en el elemento diferenciador para su elección y uso.

Mientras, los responsables aluden a que los dispositivos que más emplean son principalmente el móvil o la tablet, y en menor medida los ordenadores. Un interés que podría estar relacionado, tal y como afirman las familias, con la implementación y desarrollo que en los últimos años están adquiriendo los programas educativos con formación específica en tecnología y el soporte formativo que los Centros están ofreciendo con la toma de conciencia de los profesionales. Formación que, según los expertos entrevistados, cubre lagunas y necesidades relacionadas con la comunicación individual y grupal que precisa este colectivo y que van relacionadas con el crecimiento de la preocupación y las políticas educativas orientadas a la consecución de la plena inclusión educativa de los sujetos con discapacidad.

¿Qué influencias han tenido en las personas con discapacidad intelectual? ¿Cuáles son las barreras principales con las que se han encontrado?

Las personas con discapacidad intelectual encuentran, en muchos casos, importantes limitaciones para poder aprovechar el potencial que caracteriza a las tecnologías y el uso de las diferentes plataformas. En esta línea, los usuarios y los formadores indican que sus necesidades no siempre se tienen en cuenta al diseñar los medios tecnológicos y los recursos que aportan. "Son dificultades que se convierten en barreras de acceso y que suponen un serio inconveniente para un porcentaje importante de la población con discapacidad y que conllevan situaciones de riesgo de exclusión", comenta la psicóloga del Centro de AMI-3. Y precisamente en ello inciden otros profesionales del Centro entrevistados, quienes además aluden a que un porcentaje bastante elevado de los usuarios de su Centro presentan dificultades de lectoescritura, por lo que la utilización del recurso de voz y audio les resulta muy 
útil y de aquí que utilicen WhatsApp como el sistema más habitual de comunicación, comenta la pedagoga entrevistada.

Las barreras y las soluciones que se presentan varían, tal como señalan los profesionales y las familias entrevistadas, ya que su utilización e integración no va a depender únicamente del tipo o grado de discapacidad, sino de las dificultades encontradas en el uso y manejo de los dispositivos. Los profesionales del Centro potencian dentro de la formación impartida la adquisición de habilidades sociales y digitales, adecuando sus prácticas pedagógicas al entorno virtual a través de herramientas como plataformas de videoconferencia (Zoom) o de vídeo (WhatsApp). La facilidad de uso y su accesibilidad es lo que promueve esta selección y lo que las diferencia del resto, ya que incluyen recursos tecnológicos sencillos y fáciles de manejar: "El WhatsApp y el Zoom han sido las herramientas preferidas por los usuarios del Centro para mantener la actividad durante la pandemia", comenta la monitora del servicio de ocio entrevistada.

La utilización de las TIC en el proceso de enseñanza-aprendizaje del usuario aumenta la autoestima y motivación, lo que les ayuda a integrarse y no sentirse excluidos entre sus grupos sociales. Además, la enseñanza a través de Internet les ayuda a desarrollar habilidades cognitivas de suma importancia, comentan los psicólogos y pedagogos entrevistados. Una educación que se presenta especialmente favorable para las personas con necesidades educativas especiales tanto para la mejora de sus habilidades cognitivas como para su normalización e integración social.

¿Consideras que se encuentran adaptadas las plataformas a las necesidades de las personas con discapacidad? ¿Cuáles son las características que demandan para que sean herramientas más inclusivas?

La respuesta unánime de los profesionales entrevistados en el Centro fue que en ocasiones las herramientas no están adaptadas a las necesidades de las personas con discapacidad, ya que sus características y las funciones que ofrecen no están adaptadas, lo cual dificulta su accesibilidad y usabilidad. Destacan que la lectoescritura es una de las barreras fundamentales con las que se encuentran, ya que bastantes usuarios del Centro muestran dificultades para leer o escribir, por lo que los dispositivos y plataformas no deberían basarse principalmente en este aspecto, sino priorizar otras alternativas que permitiesen hacer los contenidos más accesibles.

Puntualizando en este aspecto la respuesta de los psicólogos fue más precisa en cuanto a que sí encuentran que existen importantes limitaciones para poder aprovechar el potencial que caracteriza a las tecnologías, debido a que no siempre se tienen en cuenta las necesidades del colectivo al diseñar estos medios y tampoco ayudan a los contenidos que a través de ellos se transmiten. 
Entre los usuarios entrevistados en el marco de esta investigación también apuntaron a que otra de las causas es la disminución del tamaño de los teléfonos móviles. Lo que indudablemente repercute en el tamaño de los teclados, los caracteres y las pantallas, resultando en muchos casos compleja la utilización de los menús y del procedimiento para enviar mensajes. Un escenario que, si bien no impiden el uso del móvil, sí que, para las personas con menor nivel de desarrollo intelectual, constituyen auténticas barreras.

$\mathrm{Y}$ en cuanto a las familias son los propios padres los que demandan que, en un futuro próximo, se pueda lograr mayor accesibilidad gracias a la creación de plataformas de interoperabilidad. Ya que no solo ayudaría a lograr un diseño más adaptado de los dispositivos móviles que actualmente se realizan, sino que se pensaría en usuarios con distintos tipos de discapacidades, lo que ayudaría a que una parte importante de la población pudiera utilizarlos adecuadamente.

\section{DISCUSIÓN Y CONCLUSIONES}

Las TIC, tal y como han puesto de manifiesto diversos estudios, pueden ser un elemento importante y de gran ayuda en la mejora de la independencia y calidad de vida de las personas con discapacidad (Acosta et al., 2020; de-Souza-Godino, 2021), así como en su inclusión educativa, social y laboral (Delgado-Ramírez et al., 2021; Puentes-Sánchez, 2020; Viñarás-Abad et al., 2021). Sin embargo, y aunque resulte paradójico, si el avance de las tecnologías no tiene en cuenta las demandas y necesidades de este colectivo, puede lograr el efecto contrario, convirtiéndose en una nueva forma exclusión social (El-Homrani et al., 2017).

Este estudio se ha centrado en analizar el uso que hacen las personas con discapacidad intelectual de las plataformas digitales y en examinar las ventajas y dificultades que han encontrado los diferentes agentes educativos a partir de la emergencia sanitaria provocada por la COVID-19, que ha impuesto la modalidad virtual como única vía de enseñanza.

Tal y como muestran los datos obtenidos, y en consonancia con la investigación de Gómez-Puerta y Chiner (2018), aunque todavía queda un largo camino para que todos los ciudadanos puedan aprovechar al completo las oportunidades y ventajas que ofrecen las TIC, cada vez son más las personas con discapacidad intelectual que las incorporan a su entorno social y acceden a Internet. Asimismo, en la línea de lo expuesto en la investigación de Arrieta-Casasola (2019), todos los agentes educativos participantes coinciden en que suponen un elemento motivador para los jóvenes con discapacidad.

De acuerdo a los hallazgos obtenidos, la pandemia de la COVID-19 ha supuesto un cambio significativo no solamente para las personas con discapacidad, sino también para los centros de formación que se han visto obligados a adaptar sus prácticas educativas, así como a intervenir en la promoción, facilitación y formación de los usuarios con discapacidad en el uso de las plataformas digitales. Además 
de a nivel comunicativo, abriendo nuevas formas de interacción, el confinamiento también les ha afectado en la forma de llevar a cabo los procesos de enseñanzaaprendizaje. A raíz de la situación de aislamiento impuesta, se ha incrementado sustancialmente el uso que hacen de las plataformas. Estos resultados coinciden con el estudio realizado por Down España (2021) en el que participaron 340 jóvenes con síndrome de Down y en el que se pone de manifiesto que el $82 \%$ de los encuestados ha adquirido aprendizajes para el uso de plataformas digitales durante el período de confinamiento y que tras aprender a usarlas el $69 \%$ las continúa empleando autónomamente. Asimismo, estas herramientas permitieron a la gran mayoría de jóvenes con Síndrome de Down mantenerse en contacto con sus pares a pesar de la situación de aislamiento y acostumbrarse al uso de este tipo de plataformas digitales y dispositivos para comunicarse y mantener relaciones personales con su entorno.

Del mismo modo, los hallazgos de nuestro estudio ponen de manifiesto que, a pesar de que al principio surgieron las mayores dificultades para los diferentes agentes educativos del centro ocupacional, la mayoría han conseguido adaptarse a estas nuevas demandas de la sociedad y las propuestas de la institución tuvieron buena acogida tanto para los usuarios como para los diferentes especialistas del centro. En línea con investigaciones recientes (Leal-Filho et al., 2021), la pandemia ha supuesto un cambio significativo en los procesos de enseñanza-aprendizaje, lo que implica consecuencias sustanciales para el personal educativo en lo referente al diseño y desarrollo de los procesos educativos (Tang et al., 2021). En este sentido, al disminuir los contactos presenciales, aumentó la necesidad de buscar alternativas que permitieran al menos mantener un contacto virtual y evitar, de este modo, el aislamiento social total de los usuarios del centro. El proceso de digitalización se llevó a cabo de forma precipitada y con carácter de urgencia, debido al repentino e imprevisto decreto del estado de alarma y sus correspondientes medidas de prevención y restricción, que obligaron tanto al profesorado como a las familias y el alumnado a adaptarse a nuevas modalidades de enseñanza y formarse en el uso de las TIC (Šucha y Gamme, 2021).

Este hecho ha supuesto, sin duda, una revolución educativa sin precedentes, que ha abierto nuevas oportunidades, fomentando de manera forzada el uso de las plataformas digitales. Sin embargo, los participantes también destacan algunas barreras y riesgos que puede conllevar el uso de estos recursos.

De este modo, se puede destacar una brecha digital que afectó a algunas familias, en relación con la falta de recursos o competencias que impedían la accesibilidad a la educación de algunos usuarios del centro, pero que de manera colaborativa y con diversos apoyos consiguieron solventar. Además, de acuerdo con las aportaciones de autores como Gómez-Puerta y Chiner (2018), los participantes de nuestro estudio también señalan la reticencia o desconfianza hacia el uso de estas plataformas por las amenazas que podría provocar el acceso a Internet y a las plataformas en este colectivo. Destacan también la necesidad imperiosa de promover la educación 
mediática de las personas con discapacidad para fomentar un uso responsable y crítico de las redes sociales.

Tal y como han puesto de manifiesto los participantes, WhatsApp es la plataforma predilecta por excelencia, seguida por YouTube. Esta última, junto con Instagram, son utilizadas por un perfil de usuario que muestra otras necesidades e intereses comunicativos, como darse a conocer o mostrarse en la red. Habitualmente, este perfil coincide con usuarios cuyas necesidades de comunicación y de acceso a la red van mucho más allá y tienen un grado de discapacidad menor. Por último, cabe mencionar que también conceden gran relevancia a Zoom, especialmente para el seguimiento de las sesiones educativas y para comunicarse con los compañeros. Estas plataformas son más usadas porque tienen unas características que les permiten utilizar el video y el audio, funciones que prefieren antes que el texto escrito, ya que algunos usuarios manifiestan dificultades de lectoescritura. De este modo, priorizan plataformas donde están más presentes las imágenes, vídeos y audios y no es imprescindible emplear el teclado, elemento que puede suponer barreras para algunos de los usuarios. En este sentido, los profesionales del Centro señalan que sería recomendable hacer plataformas y modelos de dispositivos más intuitivos, ágiles y sencillos de manejar, adaptados a sus necesidades.

Finalmente, de acuerdo con Peña-Estrada et al. (2020), destacamos la necesidad de continuar impulsando entornos educativos accesibles para las personas con discapacidad y apostar por la educación mediática para promover el uso efectivo y responsable de las plataformas digitales en este colectivo, con el fin de lograr una sociedad más equitativa, justa e inclusiva.

\section{APOYOS}

Este trabajo se ha desarrollado con el apoyo del Proyecto I+D «Youtubers e Instagrammers: La Competencia Mediática en los Prosumidores Emergentes» (RTI2018-2093303-B-Ioo), financiado por la Agencia Estatal de Investigación del Ministerio de Ciencia, Innovación y Universidades de España y el Fondo Europeo de Desarrollo Regional (FEDER).

\section{REFERENCIAS}

Acosta, M., Betún, A., Delgado, J., e Íñiguez, M. (2020). Las TIC como oportunidad para fortalecer el PEA en los estudiantes con discapacidad visual. Revista Tecnológica-Educativa Docentes 2.o, 9(1), 42-48. https://doi.org/10.37843/ rted.voi1.97

Aguaded, I., Jaramillo-Dent, D., y DelgadoPonce, Á. (Coords.) (2021). Currículum
Alfamed de formación de profesores en educación mediática: MIL (Media and Information Literacy) en la era posCOVID-19. Ediciones Octaedro.

Albalá-Genol, M. Á., y Guido, J. I. (2020). La brecha socioeducativa derivada del Covid-19: posibles abordajes desde el marco de la justicia social. Revista Latinoamericana de Estudios 
Educativos, 5o(ESPECIAL), 173-194. https://doi.org/10.48102/rlee.2020.50. ESPECIAL.101

Alfredsson-Ågren, K., Kjellberg, A., y Hemmingsson, H. (2020). Digital participation? Internet use among adolescents with and without intellectual disabilities: A comparative study. New Media \& Society, 22(12), 2128-2145. https://doi. org/10.1177/1461444819888398

Álvarez-Álvarez, C., y San-Fabián-Maroto, J. L. (2012). La elección del estudio de caso en investigación educativa. Gazeta de Antropología, 28(1), 1-12. https://doi. org/10.30827/Digibug.20644

Arce-Peralta, F. J. (2020). La transición del paradigma educativo hacia nuevos escenarios: COVID-19. CienciAmérica, 9(2), 115-119. https://doi.org/10.33210/ ca.v9i2.285

Arrieta-Casasola, A. (2019). TIC dirigidas a la superación de barreras educativas de las personas con discapacidad. Innovaciones Educativas, 21(31), 115-130. https://doi. org/10.22458/ie.v21i31.2698

Bonilla-del-Río, M., Valor-Rodríguez, L., y García-Ruiz, R. (2018). Alfabetización mediática y discapacidad: Análisis documental de literatura científica en Web of Science (WoS) y Scopus. Prisma Social, 20, 1-20. https://bit.ly/33AnzgG

Cáceres-Piñaloza, K. F. (2020). Educación virtual: Creando espacios afectivos, de convivencia y aprendizaje en tiempos de COVID-19. CienciAmérica, 9(2), 38-44. https://doi.org/10.33210/ca.v9i2.284

Corral-Ollero, D., y de-Juan-Fernández, J. (2021). La educación al descubierto tras la pandemia del COVID-19. Carencias y retos. Aularia, 1O(1), 21-28. https://bit. ly/2QXvhs5

De-Souza-Godino, S. (2021). Revisión sistemática sobre la tecnología de prendas y accesorios inteligentes para las personas con discapacidad. Perspectivas
Metodológicas, 21, 1-17. https://doi. org/10.18294/pm.2021.3433

Delgado-Ramírez, J. C., Valarezo-Castro, J. W., Acosta-Yela, M. T., y SamaniegoOcampo, R. D. L. (2021). Educación Inclusiva y TIC: Tecnologías de Apoyo para Personas con Discapacidad Sensorial. Revista Tecnológica-Educativa Docentes 2.o, 11(1), 146-153. https://doi. org/10.37843/rted.v11i1.204

Down España (2021, 29 de julio). Un estudio arroja importantes conclusiones sobre las consecuencias psicosociales de la pandemia entre los jóvenes con síndrome de Down. Down España. https://bit. ly/3zRMLGP

El-Homrani, M., Ávalos, I., y GarcíaMartínez, I. (2017). Las tecnologías de la información y la comunicación (TIC) para atender a la diversidad: Una respuesta educativa. En J. Ruiz-Palmero, J. Sánchez-Rodríguez y E. Sánchez-Rivas (Eds.), Innovación docente y uso de las TIC en educación (1-12). UMA Editorial. https://bit.ly/3bJXVUL

Expósito, C. D., y Marsollier, R. G. (2020). Virtualidad y educación en tiempos de COVID-19. Un estudio empírico en Argentina. Educación y Humanismo 22(39), 1-22. https://doi.org/10.17081/ eduhum.22.39.4214

García-Aretio, L. (2021). COVID-19 y educación a distancia digital: preconfinamiento, confinamiento y posconfinamiento. RIED. Revista Iberoamericana de Educación a Distancia, 24(1), 9-32. https://doi. org/10.5944/ried.24.1.28080

Gómez-Puerta, M., y Chiner, E. (2018). Comparación de las percepciones de los estudiantes de magisterio sobre los riesgos de internet para personas con y sin discapacidad intelectual y del desarrollo. International Journal of Developmental and Educational Psychology, 3(Esp.1), 189-198. https://doi.org/10.1706o/ ijodaep.2018.n1.v3.1253 
Karasel, N., Bastas, M., Altinay, F., Altinay, Z., y Dagli, G. (2020). Distance Education for Students with Special Needs in Primary Schools in the Period of CoVid-19 Epidemic. Propósitos y Representaciones, 8(3), e587. https://doi.org/10.20511/ pyr2020.v8n 3.587

Leal-Filho, W., Wall, T., Rayman-Bacchus, L., Mifsud, M., Pritchard, D. J., Lovren, V. O., Farinha, C., Petrovic, D. S., y Balogun, A. L. (2021). Impacts of COVID-19 and social isolation on academic staff and students at universities: A cross-sectional study. BMC Public Health, 21(1213), 1-19. https://doi.org/10.1186/s12889-02111040-Z

Martín-Blanco, J. (2021). El impacto de la pandemia de la COVID19 en los derechos humanos de las personas con discapacidad. Revista Latinoamericana en Discapacidad, Sociedad y Derechos Humanos, 4(2), 71-92. https://bit. ly/3f62Pw1

Martínez-Garcés, J., y Garcés-Fuenmayor, J. (2020). Competencias digitales docentes y el reto de la educación virtual derivado de la covid-19. Educación y Humanismo, 22(39), 1-16. https://doi.org/10.17081/ eduhum.22.39.4114

Moreno-Rodríguez, R., Tejada-Cruz, A., y Díaz-Vega, M. (Coords.) (2020). COVID-19. Educación Inclusiva $y$ Personas con Discapacidad: Fortalezas y Debilidades de la Teleeducación. La Ciudad Accesible. https://bit.ly/3ezASxG

Murillo, F. J., y Duk, C. (2020). El covid-19 y las brechas educativas. Revista latinoamericana de educación inclusiva, 14(1), 11-13. https://doi.org/10.4067/ $\underline{\text { So718-73782020000100011 }}$

Peña-Estrada, C., Vaillant-Delis,M., Soler-Nariño, O., Bring-Pérez, Y., y Domínguez-Ruiz，Y. (2020). Personas con Discapacidad y Aprendizaje Virtual:
Retos para las TIC en Tiempo de Covid-19. Revista Tecnológica-Educativa Docentes 2.o, 9(2), 204-211. https://doi. org/10.37843/rted.vgi2.165

Puentes-Sánchez, I. Y. (2020). Uso de las TIC como herramienta de inclusión social: una mirada desde la psicología [Doctoral dissertation, a Universidad de Cundinamarca] Dictum. https://bit. ly/3ubzGVR

Šucha, V., y Gamme, J. F. (2021). Humans and societies in the age of artificial intelligence. https://bit.ly/3io4I8R

Tang, Y. M., Chen, P. C., Law, K. M. Y., Wu, C. H., Lau, Y., Guan, J., He, D., y Ho, G.T.S. (2021). Comparative analysis of student's live online learning readiness during the coronavirus (COVID-19) pandemic in the higher education sector. Computers \& Education, 168, 104211. https://doi. org/10.1016/j.compedu.2021.104211

Tejada-Ríos, J. (2021). Accesibilidad de las personas con discapacidad en España y Portugal: Perspectiva jurídica. REJIE, 24, 93-114. https://doi.org/10.24310/ REJIE.2021.vi24.12140

UNESCO (2020). Policy Brief: Education during COVID-19 and beyond. United Nations. https://bit.ly/3fotdFh

Viñarás-Abad, M., Sánchez-Valle, M., y Vázquez-Barrio, T. (2021). Situación de las personas con discapacidad en el sector de la Comunicación en España: aspectos laborales, profesionales y académicos. Profesional de la información (EPI), 3o(2), 1-15. https://doi.org/10.3145/ epi.2021.mar.02

Wright, M. F., Wachs, S., y Gámez-Guadix, M. (2021). Youths' coping with cyberhate: Roles of parental mediation and family support. [Jóvenes ante el ciberodio: El rol de la mediación parental y el apoyo familiar]. Comunicar, 67, 21-33. https:// doi.org/10.3916/C67-2021-02 


\section{PERFIL ACADÉMICO Y PROFESIONAL DE LAS AUTORAS}

Mónica Bonilla-del-Río. Investigadora Predoctoral (FPU) y Doctoranda en el programa Interuniversitario de Comunicación en la línea de Educomunicación y Alfabetización Mediática (UHU). Máster Interuniversitario en Comunicación y Educación Audiovisual por la Universidad Internacional de Andalucía y la Universidad de Huelva. Máster en Educación Emocional, Social y de la Creatividad por la Universidad de Cantabria. Graduada en Magisterio en Educación Infantil por la Universidad de Cantabria. https://orcid.org/0000-0003-2476-8922

E-mail: monica.bonilla@dfilo.uhu.es

\section{DIRECCIÓN DE LA AUTORA}

Facultad de Humanidades. Pabellón 11

Campus de «El Carmen»

Avenida de las Fuerzas Armadas, s/n.

21007 Huelva

Ma Luisa Sánchez-Calero. Titular de Universidad en la Facultad de Ciencias de la Información de la UCM. Docente universitaria de Grado y Máster en el área de Redacción Periodística. Su línea de investigación se centra en el Discurso del Mensaje Periodístico. Pertenece al Grupo de Investigación de la Universidad Pompeu Fabra: Comunicación Política, Medios, y Democracia. Y es Codirectora del proyecto I+D+I Convocatoria 2019-MINECO. "Comunicación de crisis y emergencias en Social Media. Estudio de su uso en España y diseño de protocolos informativos para instituciones y medios de comunicación (COMCRISIS)" PID2019-109064GB-Ioo. https://orcid.org/0000-0002-7929-144X

E-mail: mlusaca@ccinf.ucm.es

DIRECCIÓN DE LA AUTORA

Facultad de Ciencias de la Información

Avda. Complutense, $\mathrm{s} / \mathrm{n}$

Ciudad Universitaria

28040 Madrid

Fecha de recepción del artículo: 22/05/2021

Fecha de aceptación del artículo: 01/08/2021

Fecha de aprobación para maquetación: 14/09/2021 